\title{
Observations of accretion shocks
}

\author{
David R. Ardila ${ }^{1}$ \\ ${ }^{1}$ Spitzer Science Center, Infrared Processing and Analysis Center, MS 220-6, California \\ Institute of Technology, Pasadena, CA 91125, USA \\ email: ardila@ipac.caltech.edu
}

\begin{abstract}
I review our current understanding of accretion shocks in classical $\mathrm{T}$ Tauri stars (CTTs), from a UV and X-ray perspective. The region of the accretion shock is a good candidate as a source of UV transition region lines from Li/Na-like ions, which are stronger in CTTs than in naked atmospheres. Disk gas captured by the stellar magnetic field produces a strong radiative shock upon falling on the stellar surface. Radiation from the shock creates a radiative precursor and heats the stellar surface resulting in a hot spot. Stellar and shock models indicate that unless the post-shock column is very large, it will be buried on the stellar photosphere. Models of the continuum emission produced by this configuration can roughly reproduce the observed excess spectra down to $1650 \AA$. Transition region lines in CTTs are broad, very variable, and present blueshifted, centered, and redshifted centroids. Detailed models of the line emission have so far failed to reproduce the fluxes, line shapes, and line ratios. High resolution X-ray line observations indicate the presence of larger amounts of cool plasma in CTTs with respect to WTTs. Observations of density sensitive line ratios of He-like ions suggest high plasma densities, as expected from lines originating in the accretion shock. For most stars, the interpretation of these ratios in terms of density remains equivocal due to the presence of the strong UV continuum.
\end{abstract}

Keywords. Stars: atmospheres, coronae, winds, outflows; line: formation; magnetic fields; ultraviolet: stars; X-rays: stars

\section{Introduction}

The presentations in this volume testify to the substantial progress made in our understanding of T Tauri stars since Alfred Joy first described them (Joy 1945). We now believe that the strong excesses seen in CTTs are the result of the presence of an accretion disk and its interaction with the stellar magnetic field. In particular, the excess line emission at optical wavelengths has been successfully modeled (e.g. Muzerolle et al. 1998) as being due to the presence of gas captured in the extended magnetosphere. The continuum excess is believed to be primarily due to heating of the stellar photosphere by the same infall gas (Calvet \& Gullbring 1998).

Strong continuum and line excesses are also observed at ultraviolet wavelengths, and in this sense the observational problem is conceptually similar to the optical one (Fig. 1), although the shorter wavelengths imply that the lines trace higher energy processes. As in the optical, surface fluxes are much larger than expected from a naked atmosphere. For example, using International Ultraviolet Explorer data, Johns-Krull et al. (2000) showed that the surface flux in the $\mathrm{C}$ IV resonant lines can be as much as an order of magnitude larger than the largest flux observed in Weak T Tauri stars (WTTs), main sequence dwarfs, or RS CVn stars. They also showed that the excess flux in the lines is strongly correlated with accretion rate, suggesting that the lines are powered by the accretion process.

In recent years we have also seen an explosion of new observational results at Xray wavelengths thanks to the Chandra Orion Ultradeep Project (Feigelson et al. 2002) 
and, more recently, to the XMM-Newton Extended Survey of the Taurus molecular cloud (Güdel et al. 2007). Those measurements have confirmed that CTTs have lower $L_{X} / L_{B o l}$ values than WTTs, by a factor of $\sim 2$, but harder emission. This would suggest the presence of an additional source of extinction, not accounted for in the calculation of the occulting column. However, CTTs are deficient in $L_{X} / L_{B o l}$ with respect to WTTs even at the highest energies, perhaps suggesting that a fraction CTTs emission measure has been cooled to non-detectable temperatures (Telleschi et al. 2007a). Indeed, temperature sensitive X-ray spectroscopic diagnostics reveal the presence of soft X-ray excesses in CTTs (Telleschi et al. 2007b). In addition, anomalously low line ratios of He-like ions are measured in CTTs, perhaps indicating the presence of high plasma densities (see Kastner's poster in this conference).

These observational constraints fit, at least qualitatively, with our understanding of the behavior of the accretion stream. The gas captured by the magnetosphere falls along the magnetic field lines, reaching speeds comparable to the free-fall velocity $(\sim 300 \mathrm{~km} / \mathrm{s})$. The density of the accretion stream depends on the accretion rate (see $\S 2$ ) but it is typically of the order of $\sim 10^{12} \mathrm{~cm}^{-3}$. The supersonic flow, confined by the magnetic field, produces a strong J-shock upon reaching the star and converts most of the kinetic energy of the gas into thermal energy. The gas heats to temperatures of the order of a million degrees immediately after the shock surface and it cools radiatively until it reaches the stellar photosphere. Therefore, one expects temperature diagnostics to reveal a range of temperatures ranging from a few times $10^{6} \mathrm{~K}$ down to the hot spot temperature, and density diagnostics to reveal higher densities than those found even in active stellar atmospheres $\left(10^{9} \mathrm{~cm}^{-3}\right.$ to $10^{11} \mathrm{~cm}^{-3}$, see Ness et al. 2004).

In this contribution, I will review the current understanding of UV and X-ray line and continuum observations in the context of this accretion shock model. Fundamentally, the questions are the following: what region of the T Tauri system are the UV and X-ray observations describing? Is the emission from the accretion shock region enough to explain those observations, or are other regions of the system contributing to the emission? An understanding of the radiation coming from the accretion shock and its interaction with the rest of the $\mathrm{T}$ Tauri system is crucial to map the disk photoevaporation (Alexander et al. 2004), model the heating of jets in their launching regions (Gómez de Castro \& Verdugo 2007), and interpret broad-band X-ray observations of young stars (Preibisch et al. 2005).

\section{The shock region}

The density of the gas immediately before the accretion shock is given by (Calvet \& Gullbring 1998):

$n_{\text {pre }}=\frac{\dot{M}}{\mu m_{H} f 4 \pi R_{*}^{2}}=5.8 \times 10^{12} \mathrm{~cm}^{-3}\left(\frac{\dot{M}}{10^{-8} M_{\odot} / y r}\right)\left(\frac{M}{0.5 M_{\odot}}\right)^{1 / 2}\left(\frac{R}{2 R_{\odot}}\right)^{-3 / 2}\left(\frac{f}{0.01}\right)^{-1}$

where $\dot{M}$ is the accretion rate, $\mu$ is the mean molecular mass, $m_{H}$ is the mass of a hydrogen atom, $R_{*}$ is the stellar radius and $f$ is the shock filling factor. This last parameter specifies size of the accretion shock surface on the star. After the shock, the gas density and velocity are increased and decreased by a factor of four, respectively. The kinetic energy is transferred to thermal energy and the gas temperature is given by:

$$
T_{s}=\frac{3}{16} \frac{\mu m_{H}}{k} v_{p s}^{2}=8.6 \times 10^{5} K\left(\frac{M}{0.5 M_{\odot}}\right)\left(\frac{R}{2 R_{\odot}}\right)^{-1}
$$



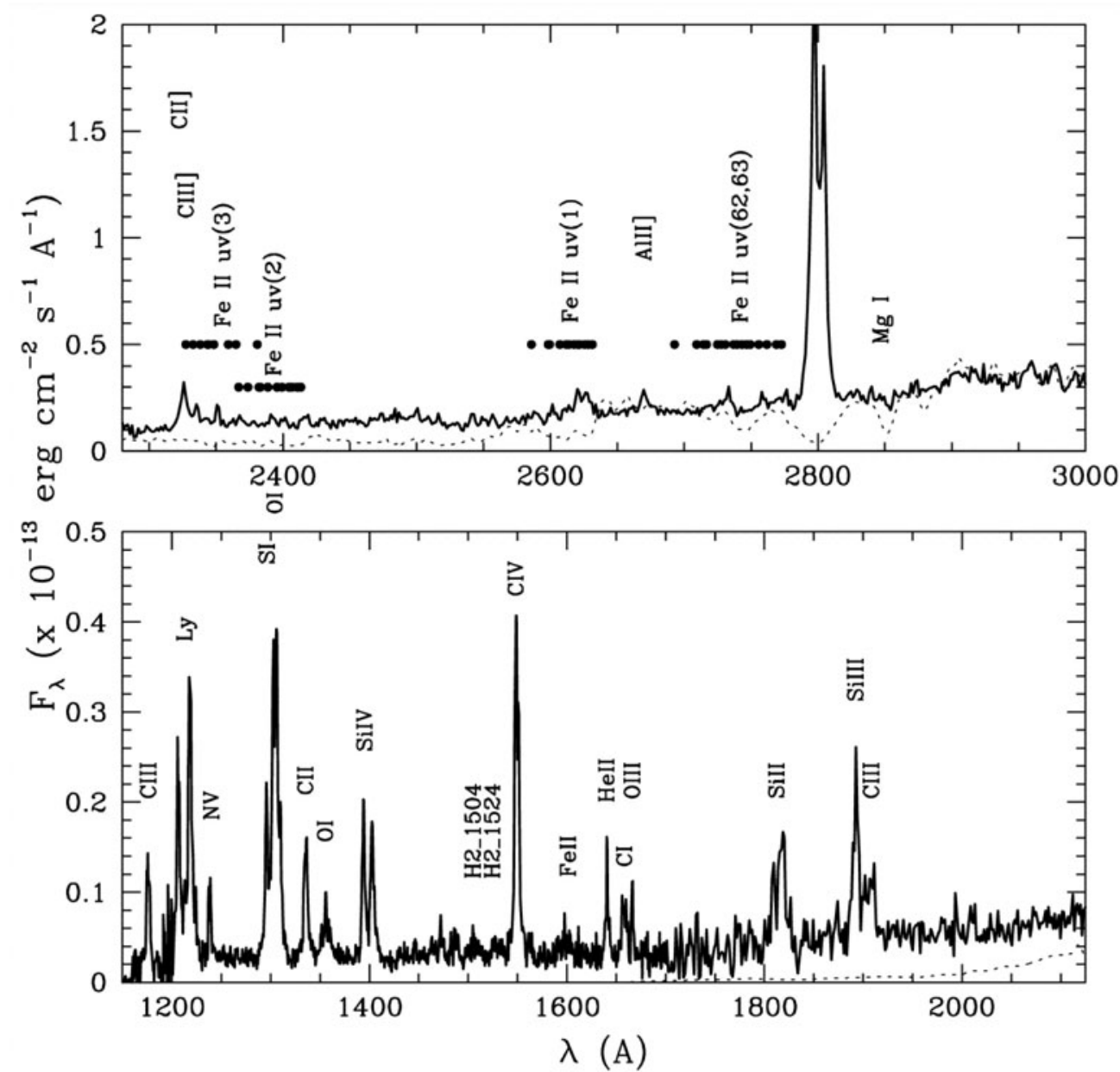

Figure 1. UV spectrum of EZ Ori, taken from Calvet et al. (2004). Top: 2270 to $3000 \AA$. Bottom: 1150 to $2120 \AA$. The dotted line is the expected emission from the naked G0 photosphere.

where $k$ is the Boltzmann constant and $v_{p s}$ is the pre-shock velocity of infall gas. The typical densities and temperatures of the gas are given in Figure 2. The post-shock gas immediately after the shock surface is hot enough to produce soft X rays $(\S 5)$ and it will cool over a distance given by the cooling length:

$$
l_{\text {post }}=\frac{3 k T v_{\text {post }}}{n \Lambda}
$$

where $\Lambda$ is the plasma cooling function. Half of the cooling radiation from the post-shock gas will be emitted toward the star, creating a hot spot with temperature of

$$
T_{e f f}=5421 K\left(\frac{\dot{M}}{10^{-8} M_{\odot} / y r}\right)^{1 / 4}\left(\frac{M}{0.5 M_{\odot}}\right)^{1 / 4}\left(\frac{R}{2 R_{\odot}}\right)^{-3 / 4}\left(\frac{f}{0.01}\right)^{-1 / 4}
$$

For some stars, the hot spot temperature can be as high 10,000 K. The other half will be emitted toward the incoming accretion flow, and it will create a radiative precursor to the shock (the "pre-shock") with typical temperatures of the order of 10,000 K. The emergent emission from the shock region is the sum of radiation emitted by optically thin gas from the pre- and post-shock, plus radiation from the heated photosphere. Within 


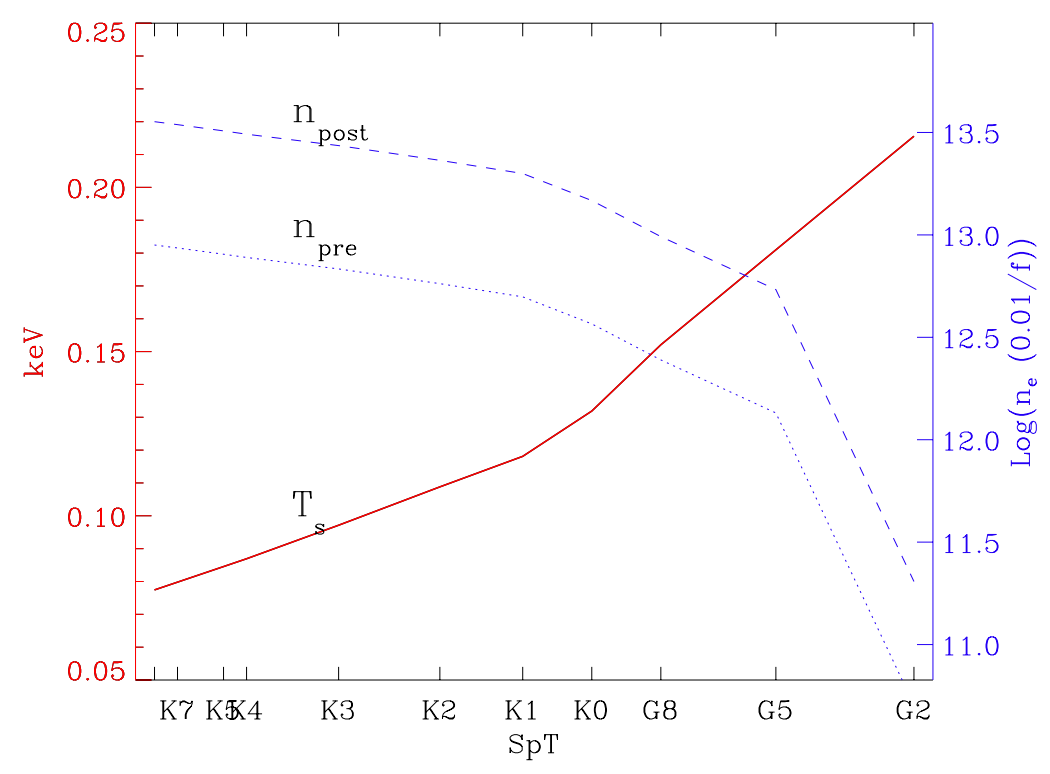

Figure 2. Gas temperature immediately after the shock (solid red) and pre- and post-shock densities (dotted green and dashed green, respectively). To make this plot I have assumed an accretion rate $\dot{M}=10^{-8} \mathrm{M}_{\odot} \mathrm{yr}^{-1}$ and the 1 Myr mass-radius relationship from D'Antona \& Mazzitelli (1997). $0.1 \mathrm{keV}$ is $1.2 \times 10^{6} \mathrm{~K}$.

this conceptual framework, Calvet \& Gullbring (1998) have attempted to reproduce the excess continuum emission observed in CTTs (lines receive a very schematic treatment in these models). Their simulations indicate that the post-shock region is very small, with depths (from the shock surface to the stellar surface) ranging from 1 to $1,000 \mathrm{~km}$, for typical parameters. The size of the pre-shock is of the order of $1,000 \mathrm{~km}$, from the shock surface to the point in which the gas becomes neutral. The models assume that all the radiation is observed with the shock surface perpendicular to the observer (face-on). Preand post-shock regions are mostly transparent to the hot spot radiation at wavelengths longer than $\mathrm{Ly}_{\alpha}$, and this hot spot radiation dominates at optical wavelengths. The contribution from the pre-shock, appearing mainly wavelengths $<3000 \AA$, becomes more important as the gravitational potential well of the star becomes larger. The continuum contribution of the post-shock emission is small in all cases.

Based on comparisons between the observed excesses and the models, Calvet \& Gullbring (1998) derive accretion rates of the order of $\dot{M}=10^{-8} \mathrm{M}_{\odot} \mathrm{yr}^{-1}$, as well as filling factors $f \sim 0.001-0.01$. The models are successful reproducing the empirical $L_{U}$ vs. $L_{a c c}$ relationship (Gullbring et al. 1998), and the gross shape of the excess spectra (Figure 3). Interestingly, the models underpredict the continuum below $1650 \AA$. Bergin et al. (2004) argue that collisionaly excited (by hot electrons) molecular hydrogen is responsible for the observed excess continuum below $1650 \AA$. One limitation of the Calvet \& Gullbring (1998) models is their assumption of coronal equilibrium in calculating the cooling function (Figure 4). The correct ionization distribution will alter the geometry of the post-shock and will result in a different emitted spectrum. 


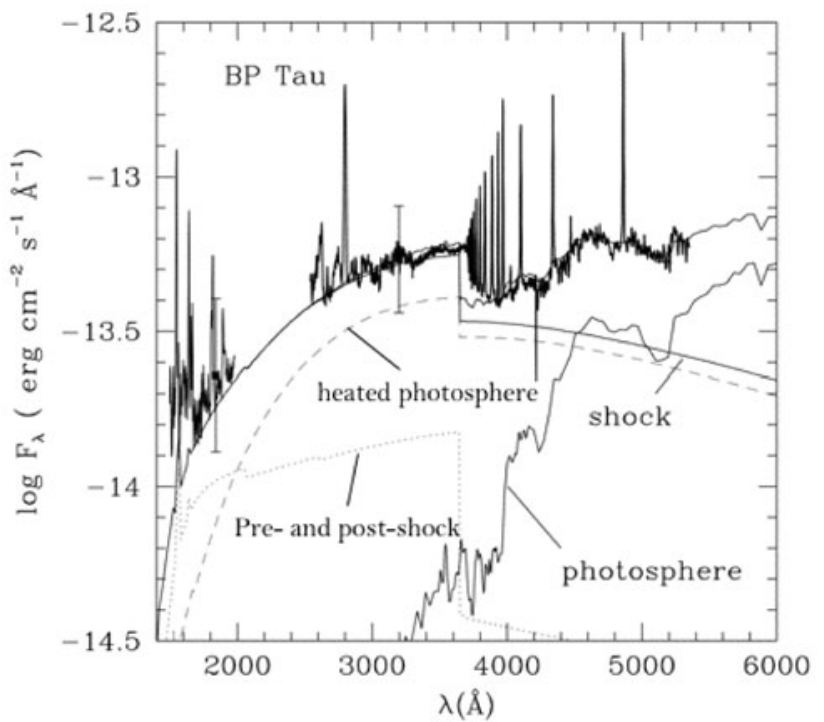

Figure 3. Results of continuum fitting to BP Tau, based on the models by Calvet \& Gullbring (1998). The observations are shown with a dark trace. The solid line ("shock") is the sum of the heated photosphere, the pre-shock, and the post-shock. Adapted from Gullbring et al. (2000).

\section{Observations of transition-region lines}

Line emission in the UV, due to atomic species, has been described in detail (e.g. Ardila et al. 2002), although the exact characteristics of the material they are tracing remains very poorly understood. Here I will concentrate primarily in the well-studied family of Lithium and Sodium-like ions (filled shells with an unpaired electron in a S orbital) whose resonant transitions are commonly seen in the UV spectra of CTTs. Examples include $\mathrm{C}$ IV $(\lambda \lambda 1548,1550 \AA)$, Si IV $(\lambda \lambda 1394,1403 \AA), \mathrm{N}$ V $(\lambda \lambda 1238,1243 \AA)$, and $\mathrm{O}$ VI $(\lambda \lambda 1032,1038 \AA)$. These are the "transition region" lines, so called because in stellar atmospheres they are produced in the region in which the temperature profile transitions from chromospheric to coronal temperatures. In ionization equilibrium, the maximum population of these ions occur at $\sim 10^{5} \mathrm{~K}$. In all these ions, if the emission comes from an optically thin, static plasma, the flux in the doublets should be in a 2:1 ratio.

As Figure 5 shows, the lines of TW Hya are fairly broad ( 200 to $\sim 300 \mathrm{~km} / \mathrm{s}, \mathrm{FWHM})$ and the bulk of their emission is redshifted. In this, they are reminiscent of $\mathrm{H}_{\alpha}$ lines. However, as it is often the case in CTTs, single observations do not do justice to the diversity of lines observed in other stars. Figure 6 shows a set of observations of the C IV doublet (Ardila et al. 2002). For comparison the lines of the WTTs HBC 388 are also shown. The lines in the WTTs are narrow $(\sim 60 \mathrm{~km} / \mathrm{s})$ and well centered on the star, and the doublet fluxes are in a 2:1 ratio. For the CTTs the lines are broad (200 to $300 \mathrm{~km} / \mathrm{s}$ ) with line ratios not always 2:1 (for example in DG Tau, DR Tau, RU Lup, and RW Aur). The line centroids vary from blue (DG Tau, RY Tau), to zero (RU Lup, BP Tau, DF Tau, T Tau), to red (upper right panel of DR Tau, TW Hya in Fig. 5). The two doublet lines from RW Aur do not look anything like each other, suggesting a steeper density or temperature profile in the emitting region, with respect to other stars.

As in other wavelength ranges, variability is a common feature of the UV lines. Multiple observations of the same target show that the lines may be strongly variable. The two observations of DR Tau shown in the top row of Figure 6 are separated by two years. Strong blueshifted emission (at $-250 \mathrm{~km} / \mathrm{s}$ ) is observed in both members of the doublet in 


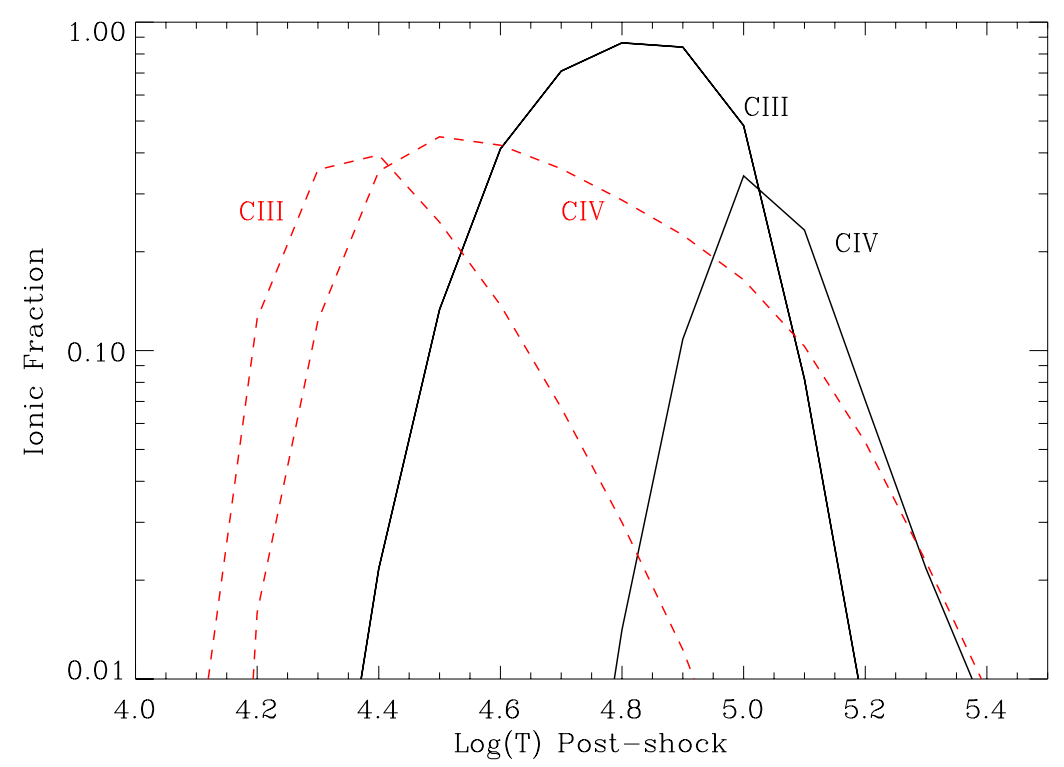

Figure 4. Given the large temperature gradient of the post-shock, the gas will not be in ionization equilibrium. The figure shows the ionization state of $\mathrm{C}$ III and $\mathrm{C}$ IV both in ionization equilibrium (solid black, from Mazzotta et al. 1998) and in the accretion shock (dashed red, from Ardila \& Johns-Krull 2007).

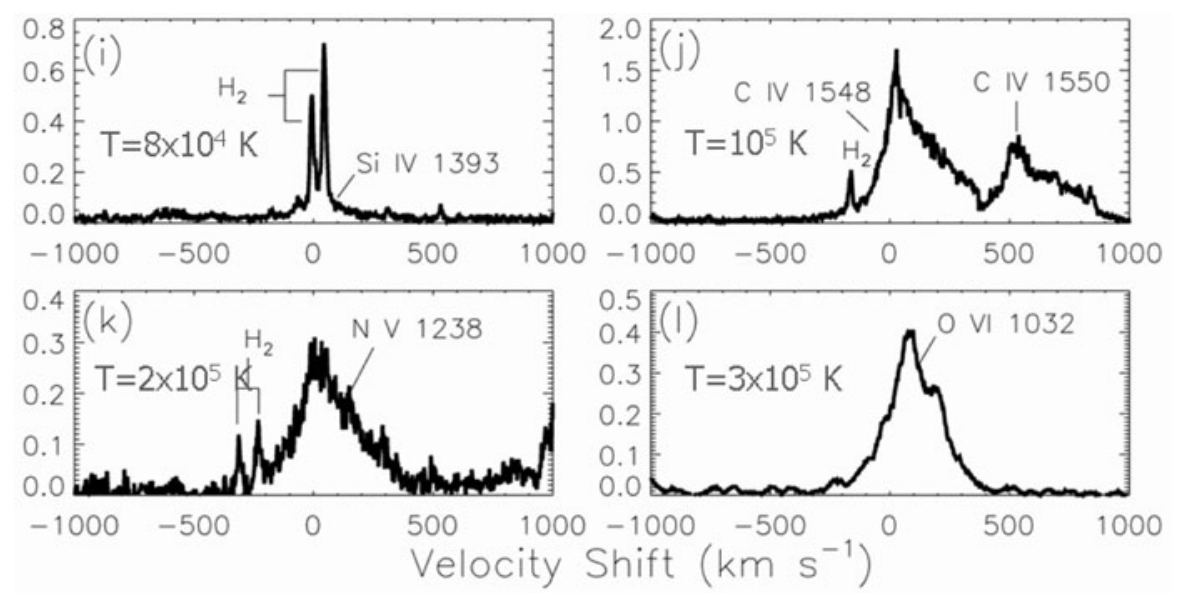

Figure 5. Examples of transition region lines in the CTTs TW Hya. For all panels, the ordinate is the flux density in units of $10^{-14} \mathrm{ergs} \mathrm{s}^{-1} \mathrm{~cm}^{-2} \AA^{-1}$, while the abscissa is the wavelength scale in $\mathrm{km} / \mathrm{s}$ centered in the stellar rest velocity. In each panel, the temperature at which the maximum population of the ion occurs under conditions of ionization equilibrium has been indicated. As is common in CTTs (Ardila et al. 2002; Herczeg et al. 2006), Si IV is buried beneath the fluorescent molecular hydrogen emission. Adapted from Herczeg et al. (2002).

the 1993 (4th top panel, from the left). This emission is absent from the 1995 observations (5th top panel, from the left), for which the DR Tau lines look similar to those of TW Hya. Figure 7 shows another example of variability, this time for RU Lup. Given the similarities in atomic parameters, one would expect all the resonant lines from $\mathrm{Li} / \mathrm{Na}-$ like ions to look similar. Indeed, the STIS 2000 observations show similarly broad, well 


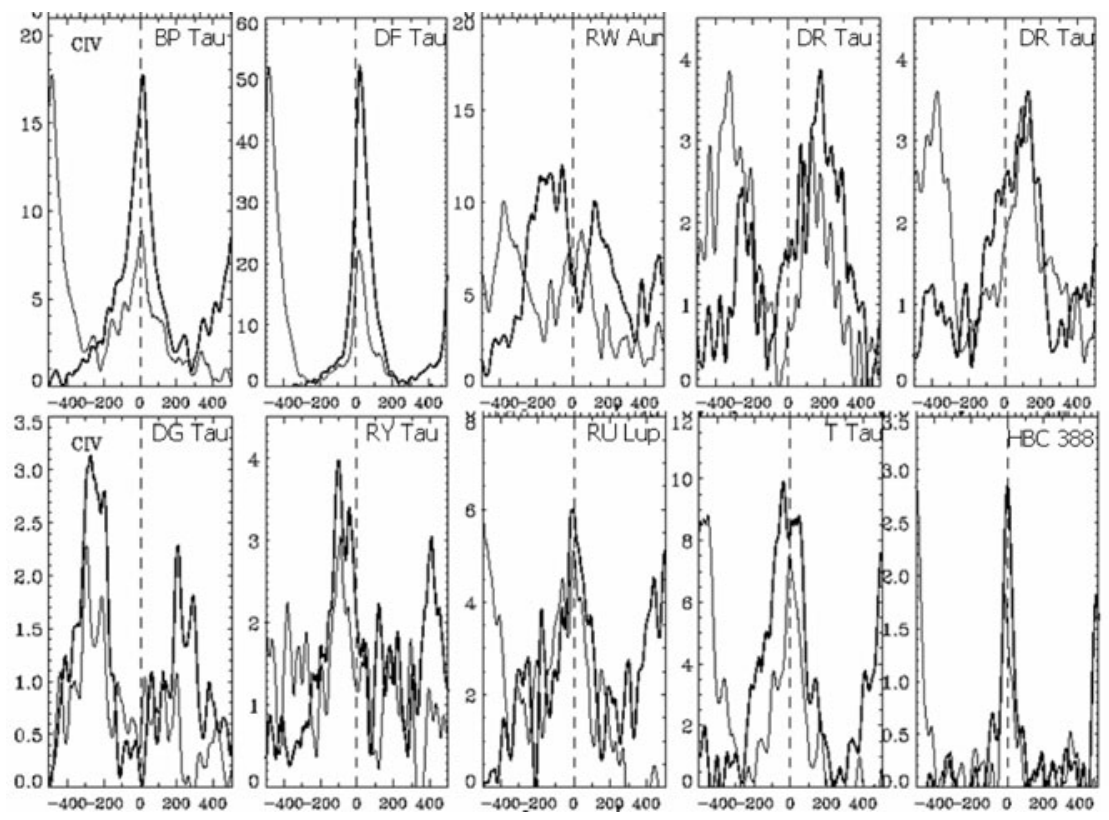

Figure 6. Examples of C IV lines in a variety of CTTs and one WTTs. The units are the same as in Figure 5. Both C IV lines are plotted in each panel, with the optically thicker member of the doublet indicated by the thicker line. The WTTs is HBC 388 (lower right) and it serves as a benchmark for the emission from a naked atmosphere. Two epochs are shown for DR Tau: 1993, 4th panel from the left on top; 1995, 5th panel from the left on top. Adapted from Ardila et al. (2002).

centered emission for C IV, Si IV, and N v. However, the FUSE 2001 observations show a very blueshifted O VI line. Herczeg et al. (2005) also show that the $\mathrm{C}$ III lines at $\lambda 1175$ $\AA$ and $\lambda 1177 \AA$, which trace the accretion flow, are strong in the first epoch, but absent in the second. They attribute this behavior to the disappearance of the accretion stream in the second epoch, perhaps leaving behind only the signature of a wind.

The accretion shock region provides a natural source of energy for these emission lines. If produced in the pre-shock, the lines should be centered at the large infall velocities (ignoring projection effects), while in the post-shock their velocities will depend on where exactly they are created, but will always be redshifted. Producing blueshifted lines in the accretion shock requires that the near accretion stream (to the observer) be absent or occulted. Alternatively, an additional line source, like an outflow, may be responsible for the blueshifted emission.

\section{Predictions of models}

The most detailed published models of line emission from the accretion shock regions of CTTs have been developed by Lamzin and collaborators (see Lamzin 2003 and references therein). These semi-analytic models consider the role of the post-shock in heating the radiative precursor and the stellar surface, and trace the optical depth of the lines in all the regions. From these models they conclude that the pre-shock should be the primary source of the transition region lines for infall velocities larger than $300 \mathrm{~km} / \mathrm{s}$. If so, one would expect to see very redshifted lines in a large fraction of the sample, but this is not what is observed. As a matter of fact, realistic attempts to explain the line shapes and centroids have so far failed (Lamzin 2003), perhaps indicating that the field lines that 


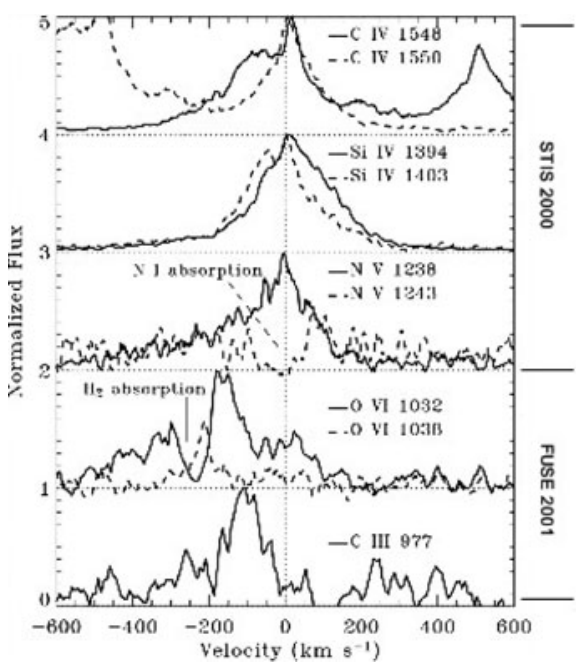

Figure 7. Variability in RU Lup. The top three panels show STIS 2000 observations of C IV, Si IV, and N V. The bottom two show FUSE 2001 observations of O VI and C III. Given the similarity in atomic parameters of the $\mathrm{Li} / \mathrm{Na}$-like iones, one would expect their shapes and centroids to be similar. Adapted from Herczeg et al. (2005).

confine the flux depart from a parallel configuration very close to the star (Lamzin 2000, 2003). The models also predict line fluxes that are 10 to 100 times larger than observed, and transition line ratios 10 times smaller than observed. At this point it is not clear if the models need to be developed further or if the lack of agreement with observations is pointing toward novel physical conditions. The contribution by S. Lamzin in this volume addresses some of these issues.

In addition to the accretion shock, other regions have been invoked as a source of the emission lines. Alexander et al. (2005) argues that photoionizing radiation with luminosities between $10^{41}$ to $10^{44}$ photons/sec is necessary to dissipate the disk and to explain the fraction of classical and weak $\mathrm{T}$ Tauri stars in star formation regions. Differential emission analysis on the UV spectra indicates that these ionizing luminosities are available in the region producing the UV lines. However, their models suggest that the flux cannot be produced in the accretion shock because it would be absorbed by the incoming accretion column (Alexander et al. 2004). Therefore, they conclude that the lines observed in UV spectra of CTTs have to come from the stellar atmosphere: they are real transition region lines. Their models assume a $1 \mathrm{R}_{\odot}$ long pre-shock column, longer than any predicted by models, and no radiation escaping from the column sides (which may be negligible anyway, see $\S 5$ ). Therefore, they overestimate the importance of the accretion column as a source of extinction. If one accepts that the UV lines are primarily atmospheric in origin this implies that the presence of accretion radically alters the physical characteristics of the stellar atmosphere, as these strong UV lines are not seen in WTTs.

Gómez de Castro \& Verdugo (2007) analyze the spectrum of RY Tau, and argue that the species with the smallest critical densities are more blueshifted than those with large critical densities, as one would expect from a wind. They do not address how can such wind be heated to the large measured temperatures $(60,000 \mathrm{~K}$, based on their C IV/Si III] line ratios). In this context, DG Tau A provides an interesting example. The star is well known to have a jet that has been seen in a variety of wavelengths, perhaps even in X-ray images (Güdel et al. 2005). Its C IV line is blueshifted by $250 \mathrm{~km} / \mathrm{s}$, which may indicate 


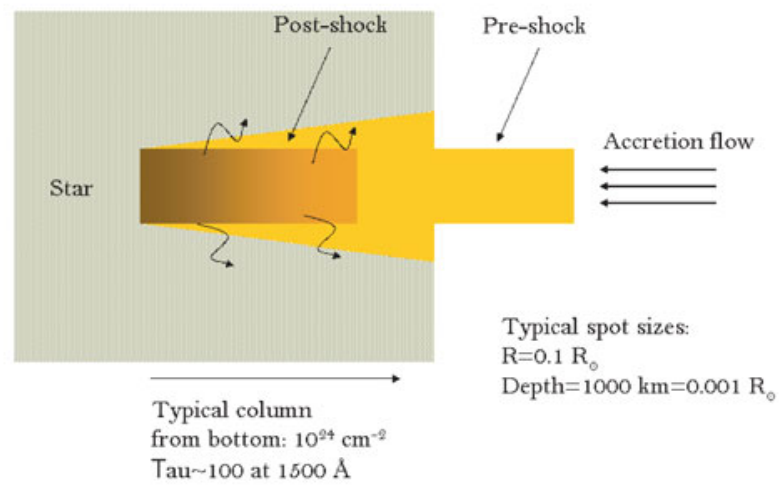

Figure 8. A cartoon of the shock region. In this cartoon, the aspect ratio of the pre- and post-shock is incorrect because for typical filling factors the shock is wider than its long.

a wind origin or a very asymmetric accretion configuration. If heated by a shock in the wind, the line should come from the radiative precursor of the shock. Wind velocities as large as $600 \mathrm{~km} / \mathrm{s}$ have been measured for DG Tau A in He I (Beristain et al. 2001).

Multiple processes may be at work here. It is possible that weak blueshifted wind emission is present in all cases but masked by redshifted accretion shock emission. Observationally, they way to attack this problem is with multi-epoch UV observations, with high enough resolution $(\mathrm{R} \sim 20,000$, like that provided by the Cosmic Origins Spectrograph - COS) to trace the line shape. As the accretion spot disappears from sight, one should see only the wind signature, if present.

\section{X-rays from shocks}

The gas immediately after the shock surface has enough thermal energy to produce soft $\mathrm{X}$-ray emission, but a large fraction of this emission will be absorbed by the pre-shock. If observed, this soft X-ray emission should primarily come from the edges of the post-shock column. As argued by Drake (2005), the position of the bottom of the post-shock column is determined by the equilibrium between the ram pressure of the incoming flow and the stellar gas pressure. For example, based on a Kurucz model of a star with $\mathrm{T}_{\text {eff }}=4250 \mathrm{~K}$, the bottom of the post-shock column in BP Tau $\left(\mathrm{p}_{\text {ram }}=7 \times 10^{3} \mathrm{dyn} / \mathrm{cm}^{2}, l_{c} \sim 1 \mathrm{~km}\right.$, Calvet \& Gullbring 1998) should be buried under a hydrogen column of a few times $10^{23} \mathrm{~cm}^{-2}$. At this column, all soft X-ray post-shock radiation will be absorbed by the stellar photosphere. For TW Hya $\left(\mathrm{p}_{\text {ram }}=3 \times 10^{3} \mathrm{dyn} / \mathrm{cm}^{2}, l_{c} \sim 1000 \mathrm{~km}\right.$, based on an accretion rate of $\dot{M}=10^{-9} \mathrm{M}_{\odot} \mathrm{yr}^{-1}$ and a post-shock length from Ardila \& JohnsKrull 2007), the column density at the bottom of the post-shock is also of the order of $10^{23} \mathrm{~cm}^{-2}$, but the post-shock is large enough that the stellar column density at the shock surface is two orders of magnitude less. Therefore, radiation from the post-shock may escape from the sides of the column and be observed (Figure 8). In other words, X-ray emission should only be observed in cases when the flow has low ram pressures (low accretion rate, low infall velocity, or very large spot sizes).

X-ray observations are roughly consistent with this picture. As indicated in Figure 9, XMM-Newton/EPIC observations reveal that TW Hya has a strong soft X-ray excess, compared to other CTTs. Günther et al. (2007) has modeled EPIC observations of TW Hya, by predicting the soft X-ray emission from the post-shock column. Their models 


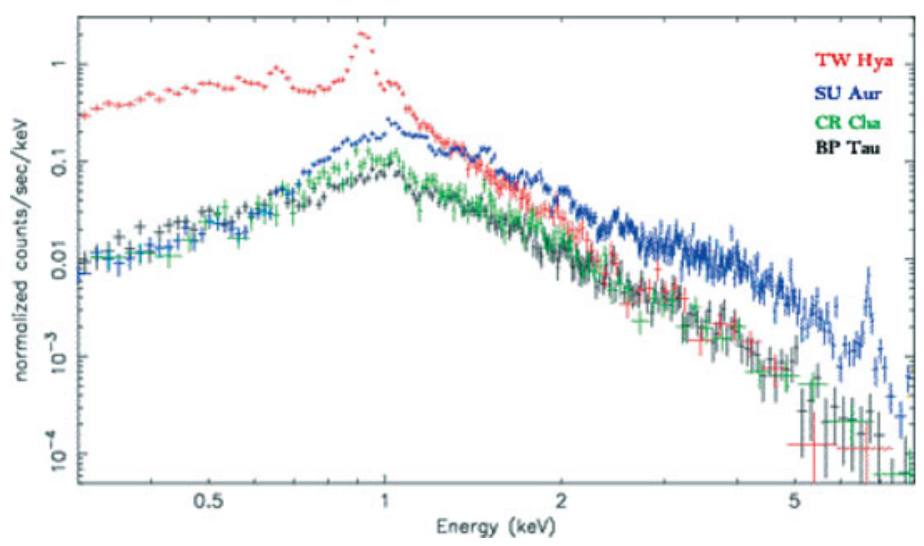

Figure 9. XMM-Newton/EPIC observations of four CTTS, adapted from Robrade \& Schmitt (2006).

consider only emission from the post-shock: no absorption due to pre-shock or stellar atmosphere. They can reproduce the observed spectral shape with a three-component spectrum that includes, in addition to the shock emission, plasmas with temperatures of $3 \times 10^{6} \mathrm{~K}$ and $16 \times 10^{6} \mathrm{~K}$. From their models, they derive $\dot{M}=10^{-10} \mathrm{M}_{\odot} \mathrm{yr}^{-1}$ and $f=0.2-0.4 \%$. The fit is marginally better than the one obtained in a simple threeplasma component model (Stelzer \& Schmitt 2004). The values of the accretion rate are one order of magnitude lower than those derived from optical and UV observations (Alencar \& Batalha 2002; Herczeg et al. 2004), which may point to the fact that the shock column is indeed partially buried. The model has also been applied to observations of V4046 Sgr (Günther \& Schmitt 2007).

As Figure 9 shows, strong soft excess is not as noticeable in other CTTs. However, these kind of low-resolution observations are not very sensitive to $<0.2 \mathrm{keV}$ emission. Measurements of the temperature sensitive $\mathrm{O}$ VII/O VIII line ratio (tracing gas between $2 \times 10^{6} \mathrm{~K}$ and $4 \times 10^{6} \mathrm{~K}$ ) suggest that as a group CTTs have larger amounts of cool gas than WTTs (Telleschi et al. 2007a) as expected from the accretion shock. If the emission is due to post-shock gas, the density of this cool plasma should be larger in CTTs, compared to the same emission in naked atmospheres $\left(\sim 10^{9} \mathrm{~cm}^{-3}\right.$ to $\sim 10^{11} \mathrm{~cm}^{-3}$, see Ness et al. 2004). Observations of density-sensitive forbidden (for) and intercombination (int) lines of He-like ions (like Ne IXand O VII) reveal for/int values smaller than $\sim 0.5$ in CTTs, suggesting plasma densities larger than $\sim 10^{11} \mathrm{~cm}^{-3}$ (see for example Kastner et al. in this volume, and references therein).

However, questions remain as to the interpretation of these values. For example, for for/int in $\mathrm{O}$ VII for $\mathrm{T}$ Tau is $>4$, indicating low plasma densities, unlike what is expected for CTTs (Güdel et al. 2007). In addition, the forbidden line of He-like ions is depopulated by ultraviolet radiation, which will lead to overestimating the plasma density. To circumvent this problem, Ness \& Schmitt (2005) have used observations of Fe XVII line ratios in TW Hya. They find very low ratios, indicative of higher than atmospheric densities. Unfortunately, the constraints on density are ambiguous because the theoretical calculations do not cover the full range of interactions between the levels involved. One additional problem is that all the density predictions are based on ionization equilibrium calculations, which will not be valid for the post-shock. This effect remains to be fully explored. 


\section{Summary}

Disk gas captured by the stellar magnetic field produces a strong radiative shock upon falling on the stellar surface. The gas reaches temperatures $\sim 10^{6} \mathrm{~K}$ immediately after the shock surface and it cools radiatively until it reaches the star. Radiation from the shock creates a radiative precursor $(\sim 10,000 \mathrm{~K})$ and it heats the stellar surface resulting in a hot spot $(5,000$ to $10,000 \mathrm{~K})$. Stellar and shock models indicate that unless the post-shock column is very large, it will be buried on the stellar photosphere.

Models of the continuum emission produced by this configuration reveal that most of the excess veiling in a CTTs is due to hot spot radiation, with the emission from the rest of the column (pre- and post-shock) becoming important as the gravitational potential well of the star increases. Such models can reproduce the observed excess spectra down to $1650 \AA$. For shorter wavelengths, continuum emission due to molecular hydrogen in the disk has been proposed as a continuum source.

Given the range of temperatures present, the region of the accretion shock is an excellent candidate to explain the observations of UV transition region lines from Li/Na-like ions such as C IV, Si IV, O VI, and N v. Transition region lines in CTTs tend to be broad (200 to $300 \mathrm{~km} / \mathrm{s}$ ) and present blueshifted, centered, and redshifted centroids. Repeated observations of the same target reveal that the centroids may shift substantially in a timescale of years.

Detailed models of the line emission have so far failed to reproduce the fluxes, line shapes, and line ratios. It is not clear if the models need further development or if these failures are pointing toward a different shock configuration. In particular, the blueshifted emission from RY Tau and DG Tau may come, not from the accretion shock, but from a wind.

Low resolution X-ray observations indicates strong soft emission from TW Hya, and detailed shock models have been successful modeling the spectra. High resolution X-ray line observations indicate the presence of larger amounts of cool plasma in CTTs with respect to WTTs. Observations of density sensitive line ratios of He-like ions suggest high plasma densities, as expected from lines originating in the accretion shock. However, the interpretation of the commonly used line ratios is rendered equivocal by the strong UV continuum of the hot spot. Fe XVII line ratios may alleviate this problem and at least in the case of TW Hya, they confirm the high plasma densities derived from other diagnostics.

The COS instrument, to be installed on-board HST in 2008, will provide us with new UV spectroscopic data, which will help clarify the exact contribution of the accretion shock to the UV lines. The synergy of the X-ray facilities with $H S T$ will provide new insights and new mysteries, insuring, for years to come, the presence of a strong community of researchers interested in these complex, amazing objects, the T Tauri stars.

\section{Acknowledgements}

I would like to thank the organizers of the IAU Symposium 243 for the invitation to Grenoble and for a wonderful conference. I would also like acknowledge Christopher Johns-Krull and Gregory Herczeg, who provided clarity and encouragement for this work.

\section{References}

Alencar, S. H. P. \& Batalha, C. 2002, ApJ, 571, 378

Alexander, R. D., Clarke, C. J., \& Pringle, J. E. 2004, MNRAS, 354, 71

Alexander, R. D., Clarke, C. J., \& Pringle, J. E. 2005, MNRAS, 358, 283

Ardila, D. \& Johns-Krull, C. M. 2007, in preparation. 
Ardila, D. R., Basri, G., Walter, F. M., Valenti, J. A., \& Johns-Krull, C. M. 2002, ApJ, 566, 1100

Bergin, E., et al. 2004, ApJL, 614, L133

Beristain, G., Edwards, S., \& Kwan, J. 2001, ApJ, 551, 1037

Calvet, N. \& Gullbring, E. 1998, ApJ, 509, 802

Calvet, N., Muzerolle, J., Briceño, C., Hernández, J., Hartmann, L., Saucedo, J. L., \& Gordon, K. D. 2004, AJ, 128, 1294

D’Antona, F. \& Mazzitelli, I. 1997, MemSAI, 68, 807

Drake, J. J. 2005, in ESA Special Publication, Vol. 560, ESA Special Publication, ed. F. Favata \& et al., 519

Feigelson, E. D., Broos, P., Gaffney, III, J. A., Garmire, G., Hillenbrand, L. A., Pravdo, S. H., Townsley, L., \& Tsuboi, Y. 2002, ApJ, 574, 258

Gómez de Castro, A. I. \& Verdugo, E. 2007, ApJL, 654, L91

Güdel, M., Skinner, S. L., Briggs, K. R., Audard, M., Arzner, K., \& Telleschi, A. 2005, ApJL, 626, L53

Güdel, M., Skinner, S. L., Mel'Nikov, S. Y., Audard, M., Telleschi, A., \& Briggs, K. R. 2007, A\&A, 468, 529

Gullbring, E., Calvet, N., Muzerolle, J., \& Hartmann, L. 2000, ApJ, 544, 927

Gullbring, E., Hartmann, L., Briceno, C., \& Calvet, N. 1998, ApJ, 492, 323

Günther, H. M. \& Schmitt, J. H. M. M. 2007, MemSAI, in press

Günther, H. M., Schmitt, J. H. M. M., Robrade, J., \& Liefke, C. 2007, A\&A, 466, 1111

Herczeg, G. J., Linsky, J. L., Valenti, J. A., Johns-Krull, C. M., \& Wood, B. E. 2002, ApJ, 572, 310

Herczeg, G. J., Linsky, J. L., Walter, F. M., Gahm, G. F., \& Johns-Krull, C. M. 2006, ApJS, 165,256

Herczeg, G. J., et al. 2005, AJ, 129, 2777

Herczeg, G. J., Wood, B. E., Linsky, J. L., Valenti, J. A., \& Johns-Krull, C. M. 2004, ApJ, 607, 369

Johns-Krull, C. M., Valenti, J. A., \& Linsky, J. L. 2000, ApJ, 539, 815

Joy, A. H. 1945, Contributions from the Mount Wilson Observatory / Carnegie Institution of Washington, 709, 1

Lamzin, S. A. 2000, Astron. Rep., 44, 323

Lamzin, S. A. 2003, Astron. Rep., 47, 498

Mazzotta, P., Mazzitelli, G., Colafrancesco, S., \& Vittorio, N. 1998, A\&AS, 133, 403

Muzerolle, J., Calvet, N., \& Hartmann, L. 1998, ApJ, 492, 743

Ness, J.-U., Güdel, M., Schmitt, J. H. M. M., Audard, M., \& Telleschi, A. 2004, A\&A, 427, 667

Ness, J.-U. \& Schmitt, J. H. M. M. 2005, A\&A, 444, L41

Preibisch, T., et al. 2005, ApJS, 160, 401

Robrade, J. \& Schmitt, J. H. M. M. 2006, A\&A, 449, 737

Stelzer, B. \& Schmitt, J. H. M. M. 2004, A\&A, 418, 687

Telleschi, A., Güdel, M., Briggs, K. R., Audard, M., \& Palla, F. 2007a, A\&A, 468, 425

Telleschi, A., Güdel, M., Briggs, K. R., Audard, M., \& Scelsi, L. 2007b, A\&A, 468, 443 